\title{
Motivos para participar en competiciones de resistencia en corredores urbanos de Chile
}

\author{
Reasons of Chilean urban runners for participating \\ in endurance running competitions
}

\section{Motivos para participar em competiçóes de resistência em corredores urbanos do Chile}

\author{
Fernando Maureira
}

Universidad de Chile

\begin{abstract}
Resumen: Barrios y Cardozo (2002) elaboraron un cuestionario de 23 preguntas relacionadas con los motivos para participar en carreras urbanas. Ellos encontraron que los motivos de corredores en Cuba se relacionaban con el logro de metas personales, la comprobación de su aptitud física, satisfacción, etc. El objetivo de la presente investigación fue conocer los motivos que llevan a corredores urbanos chilenos a participar en carreras de resistencia y describir cómo influyen las variables de sexo, nivel de estudio, experiencia competitiva y edad sobre esas motivaciones. La muestra estuvo constituida por 533 corredores urbanos, de los cuales el 66,2\% son hombres y el 33,8\% son mujeres. En relación a la edad, el 19,1\% corresponde a menores de 18 ańos, el 68,7\% tiene entre 18 y 40 ańos y el 12,2\% son mayores de 40 ańos. Los resultados muestran que en ambos sexos y en todas las edades los motivos principales son cumplir la meta que me tracé y competir contra mi propia marca, los cuales tienen que ver con metas de logro personal. No se observan diferencias entre ambos sexos, las edades, la experiencia competitiva o el nivel de estudios en los motivos de participación en este tipo de eventos. Palabras claves: Motivación, carreras de resistencia, metas, logro personal. Abstract: Barrios and Cardozo (2002) elaborated a questionnaire of 23 questions related to the motives for practicing urban careers. They thought that the motives of corridors in Cuba were relating to the achievement of personal goals, to verify his physical aptitude, satisfaction, etc. The objective of the present investigation was to know the motives that lead to urban Chilean corridors to taking part in careers of resistance and to describe how they influence the variables of sex, level of study, competitive experience and age, on these motivations. The sample was constituted by 533 urban corridors, of which $66,2 \%$ is men and $33,8 \%$ they are women. In relation to the age, $19,1 \%$ corresponds to 18 -year-old minors, $68,7 \%$ has between
\end{abstract}

18 and 40 years and $12,2 \%$ they are major of 40 years. The results show that in both sexes and in all the ages the principal motives are to fulfill the goal that I me planned and to compete against my own brand, where they have to see both with the goals of personal achievement. Differences are not observed between both sexes, the ages, the competitive experience or level of studies in the motives of participation in this type of events.

Keywords: Motivation, careers of resistance, goals, personal achievement. Resumo: Barrios e Cardozo (2002) elaboraram um questionário de 23 perguntas relacionadas com os motivos para participar em corridas urbanas. Os autores identificaram que os motivos dos corredores de Cuba se relacionavam com o alcance de objectivos pessoais, com a comprovação da sua aptidâo física, satisfação, etc. O objectivo da presente investigação foi conhecer os motivos que levam os corredores urbanos chilenos a participar em corridas de resistência e descrever como influem as variáveis sexo, nível de estudos, experiência competitiva e idade sobre essas motivaçôes. A amostra foi constituída por 533 corredores urbanos, dos quais $66,2 \%$ eram homens e 33,8\% mulheres. Relativamente à idade, 19,15 corresponde a menores de 18 anos, $68,7 \%$ tinha entre 18 e 40 anos e $12,2 \%$ eram maiores de 40 anos. Os resultados mostraram que em ambos os sexos e em todas as idades os motivos principais são cumprir o objectivo que tracei e competir contra a minha própria marca, os quais se relacionaram com objectivos de realizaçáo pessoal. Não se observaram diferenças entre ambos os sexos, idades, experiência competitiva ou nível de estudos nos motivos de participação neste tipo de eventos.

Palavras-chave: Motivação, corridas de resistência, objectivos, realização pessoal.

\section{Introducción}

En las últimas décadas mucha gente ha centrado su atención e interés por participar en las carreras de larga distancia, lo que se ve reflejado en un gran número de maratones úrbanas

Dirección para correspondencia [Correspodence address]: Fernando Maureira. Facultad de Humanidades y Educación. Universidad Internacional SEK. Santiago de Chile. E-mail: maureirafernando@yahoo. en países como España, Argentina, Colombia, Chile, Estados Unidos, Canadá, Suiza, Alemania, Suecia, etc. En Chile el running (actividad de correr en forma individual o en grupo que incluye desde personas que buscan estar en forma hasta deportistas profesionales) se ha masificado considerablemente, tanto así que las estadísticas establecen un aumento en el número de participantes en este tipo de actividad alrededor de un $600 \%$ al cabo de pocos años, como lo muestra el índice 
de inscritos al maratón Internacional de Santiago con 1.000 participantes el año 2006, 6.000 el 2007 y más de 12.000 el 2008 (Cauas, 2008).

Este fenómeno ha llevado a estudiar el tema del running desde diversas perspectivas, incluyendo el estado psicológico antes de un maratón (Buceta, López, Pérez, Vallejo y Del Pino, 2003), la intervención psicológica en corredores de maratón (Buceta, López, Pérez, Vallejo y Del Pino, 2002) y los motivos de participación en la actividad física (Garita, 2006). En relación con la motivación para participar en competencias de resistencia, el trabajo de Ogles, Masters y Richardson (1995) muestra que las mujeres prefieren carreras más cortas, donde sus principales motivos para realizar esta actividad son la preocupación por su peso, la afiliación, la autoestima y significado de la vida. Por su parte, los varones prefieren carreras más largas, impulsados por motivaciones de logro y reconocimiento.

Barrios (2001) estudió los motivos hacia la práctica de ejercicio físico, indagando en motivos para incorporarse y mantenerse practicando carreras de diversas distancias. En su trabajo destaca que es el interés por el deporte el principal motivo de participación y no la salud, como se había mostrado en la literatura internacional. Asimismo reporta el logro de metas personales, la interacción social, el interés por el evento y la autoestima como los principales motivos de corredores para inscribirse en las competencias urbanas. Por su parte, Clough, Shepherd y Maughan (1989) estudiaron los motivos para participar en carreras recreativas, dando como resultado diferentes categorías, entre ellas destacan bienestar, social, estado físico, fitness, salud y adicción por el deporte.

Barrios y Cardozo (2002) elaboraron un cuestionario de 23 preguntas relacionadas con los motivos para participar en carreras urbanas. Este cuestionario fue aplicado a una muestra de 148 participantes que compitieron en la maratón de La Habana de 1999, concluyéndose que los motivos que llevan a los sujetos a participar de la competencia son logro de meta personal, comprobar aptitud física, satisfacción, interacción social, autoestima, sentido de la vida, atracción por la competencia e interés por el deporte.

Este mismo cuestionario fue utilizado por Llopis y Llopis (2006), quienes analizan las razones por las cuales los corredores aficionados participan en competencias de resistencia y la influencia de variables como edad, género, nivel de estudios, etc., sobre esas razones. Los análisis dan cuenta que el principal motivo es la satisfacción que produce correr, además se constatar la influencia que tiene sobre los motivos la pertenencia a un club de atletismo y poseer un entrenador personal.

En Chile no existen investigaciones relacionadas con este tema, por lo cual este estudio busca establecer los motivos por los cuales los corredores aficionados chilenos participan de las competencias masivas de resistencia. También pretende describir cómo influyen variables como el género, la edad, la experiencia competitiva y el nivel de estudios sobre las motivaciones que los impulsan a participar.

\section{Material y método}

Muestra: Estuvo constituida por 533 corredores urbanos participantes de competencias masivas y populares (con más de 10.000 participantes, tanto amateur como profesionales) de 3, 5, 8, 10 y 12 kilómetros realizadas en la Región de Valparaíso, Región Metropolitana (Santiago, Vitacura, Puente Alto) y Región del Libertador General Bernardo O’Higgins. Del total de la muestra, el $66,2 \%$ son hombres y el $33,8 \%$ son mujeres. En relación a la edad de los corredores, el 19,1\% corresponde a menores de 18 ańos, el $68,7 \%$ de los participantes de esta muestra tiene entre 18 y 40 ańos y los mayores de 40 ańos corresponden al 12,2\%. En cuanto a la experiencia competitiva (haber corrido al menos en una competencia) un $55,6 \%$ manifiesta tenerla y el $44,4 \%$ de los corredores encuestados revela que se enfrentan por primera vez a dicha situación. Del total de 296 corredores que han participado en alguna carrera 147 (49,7\%) dicen haber participado en estas competencias entre 1 y 3 años, 66 (22,3\%) entre 3 y 10 años y $83(28 \%)$ más de 10 años. Para la variable nivel de estudios, la distribución porcentual es la siguiente: sólo estudios básicos (6-13 años) $0,7 \%$, estudios secundarios $24,3 \%$ (14-18 años) y estudios universitarios (desde 18 años) un $75 \%$.

Instrumentos: Para la recopilación de información se utilizó una escala de 23 ítems elaborado por Barrios y Cardozo (2002), consistente en diferentes razones para participar en carreras de resistencia. La escala es de tipo Likert, con cinco opciones de respuesta. Sin embargo, la pregunta número 22 fue descartada por poder prestarse a confusiones y no ser aplicable a la realidad nacional. Este instrumento presentó un alfa de Cronbach de 0,894. En tanto, la prueba KMO reveló un valor de 0,856 y el test de esfericidad de Bartlett un 0,000. El análisis factorial entrega la existencia de 5 factores que explican el 60,214\% de la varianza. Para los datos de edad, sexo, nivel de estudios y experiencia competitiva se aplicó una encuesta sociodemográfica elaborada para ese fin.

Procedimiento: La recolección de datos fue realizada en el Tour IND Rancagua, la Media maratón de Viña del Mar, el Tour IND Santiago, la Corrida de fiestas patrias en la comuna de Puente Alto y en el Brooks Smart Running Tour en Vitacura. Las encuestas fueron realizadas una vez terminadas las carreras.

Análisis de datos: Se utilizó el programa estadístico SPSS versión 16.0 para windows. Se aplicaron las pruebas estadísticas descriptivas, pruebas $t$ y ANOVAs. 


\section{Resultados}

A continuación se presenta la tabla $n^{\circ} 1$ con los resultados obtenidos acerca de los motivos que tienen los corredores encuestados para participar en competencias de resistencia, mostrando el orden de prioridades según la media aritmética del grado de importancia atribuida del cuestionario.

Tabla 1. Motivos ordenados según importancia para los corredores urbanos

\begin{tabular}{|c|c|c|}
\hline Ítems ordenando según la media de las respuestas & Media & Desv. típ. \\
\hline 15. Cumplir la meta que me tracé (Logro de meta personal) & 4,51 & 801 \\
\hline 16. Competir contra mi propia marca (Logro de meta personal) & 4,38 & 988 \\
\hline 8. Divertirme durante toda la competencia (Satisfacción) & 4,30 &, 865 \\
\hline 6. Sentirme atraído por el deporte (Interés por el deporte) & 4,20 & 906 \\
\hline 23. Comprobar mi condición física (Aptitud física) & 4,16 & ,894 \\
\hline 7. Sentir el placer de correr (Satisfacción) & 4,15 & ,984 \\
\hline 1. Sentirme orgulloso de mi mismo (Autoestima) & 4,11 & ,956 \\
\hline 5. Demostrar mi interés por el deporte (Interés por el deporte) & 4,08 & 1,047 \\
\hline 17. El atractivo que tienen las competencias (Atracción por la competencia) & 3,86 & 1,077 \\
\hline 2. Sentirme más seguro de mi mismo (Autoestima) & 3,84 & 1,118 \\
\hline 4. Darle ocupación a mi tiempo libre (Sentido de la vida) & 3,82 & 1,144 \\
\hline 3. Hacer mi vida más significativa (Sentido de la vida) & 3,79 & 1,139 \\
\hline 10. Sentirme parte del grupo de corredores (Interacción social) & 3,43 & 1,230 \\
\hline 9. Encontrarme con otros corredores (Interacción social) & 3,33 & 1,266 \\
\hline 18. El prestigio que tiene esta competencia (Atracción por la competencia) & 3,24 & 1,251 \\
\hline 21. Ser parte de mi preparación para otro deporte (Conveniencia) & 3,18 & 1,335 \\
\hline 12. Que se sientan orgullosos de mí (Búsqueda de reconocimiento) & 3,16 & 1,346 \\
\hline 11. Que me vean compitiendo mis amigos y familiares (Búsqueda de reconocimiento) & 2,87 & 1,350 \\
\hline 14. Obtener mejor tiempo que otro compañero (Competición) & 2,45 & 1,353 \\
\hline 13. Vencer a otros compañeros (Competición) & 2,44 & 1,370 \\
\hline 20. Deseo de ser seleccionado y representar a mi país (Conveniencia) & 2,34 & 1,416 \\
\hline 19. Deseo de obtener algún estímulo material (Conveniencia) & 1,96 & 1,131 \\
\hline Número válido (según lista) & & \\
\hline
\end{tabular}

En la parte superior de la tabla, el primer lugar es el motivo cumplir la meta que me tracé, pregunta 15 (P15), seguido por competir contra mi propia marca (P16), en tercer lugar aparece divertirme durante toda la competencia (P8), seguido por sentirme atraido por el deporte (P6). Los dos primeros motivos pertenecen a la categoría de logro de meta personal, mientras que el tercer y cuarto motivo corresponde a las categorías satisfacción e interés por el deporte respectivamente.

En la parte final de la tabla aparecen motivos menos importantes para la gente: deseo de ser seleccionado y representar a mi pais (P20), seguido por deseo de obtener algún estímulo material (P19), ambos pertenecientes a la categoría de conveniencia.

Según las categorías a las que pertenece cada motivo, y ordenadas según importancia, en primer lugar se tiene el lo- gro de meta personal, seguida por satisfacción y, en tercer lugar, aptitud fisica. En la parte media se encuentran las categorías autoestima, sentido de la vida y atracción por el deporte, mientras que las categorías menos importantes son búsqueda de reconocimiento; conveniencia y competición.

\section{Motivos para participar en competencias de resistencia intergrupos}

En la tabla 2 se presenta un resumen de los principales motivos para participar en competencias de resistencia por corredores urbanos chilenos según su sexo, edad, escolaridad, experiencia y distancia de carrera. 
Tabla 2. Principales motivos para participar en competencias de resistencia.

\begin{tabular}{|c|c|}
\hline Categoría & Motivos \\
\hline \multicolumn{2}{|l|}{ Sexo } \\
\hline Mujeres & Cumplir la meta que me trace $(\mathrm{X}=4,59 ; \mathrm{d} s=0,758)$ \\
\hline Hombres & Cumplir la meta que me trace $(\mathrm{X}=4,48 ; \mathrm{ds}=0,827)$ \\
\hline \multicolumn{2}{|l|}{ Edad } \\
\hline Menores de 18 años & Cumplir la meta que me trace $(X=4,58 ; d s=0,724)$ \\
\hline $18-40$ años & Cumplir la meta que me trace $(\mathrm{X}=4,52 ; \mathrm{d} s=0,042)$ \\
\hline Más de 40 años & Cumplir la meta que me trace $(\mathrm{X}=4,37$; $\mathrm{d}=0,809)$ \\
\hline \multicolumn{2}{|l|}{ Escolaridad } \\
\hline Estudios secundarios (14-18 años) & Cumplir la meta que me trace $(\mathrm{X}=4,59 ; \mathrm{d} s=0,751)$ \\
\hline Estudios universitarios (más de 18 años) & Cumplir la meta que me trace $(\mathrm{X}=4,45 ; \mathrm{d} s=0,809)$ \\
\hline \multicolumn{2}{|l|}{ Experiencia } \\
\hline Sin experiencia & Cumplir la meta que me trace $(X=4,36 ; d s=0,934)$ \\
\hline 1-3 años & Cumplir la meta que me trace $(\mathrm{X}=4,51 ; \mathrm{d} s=0,720)$ \\
\hline 3-10 años & Cumplir la meta que me trace $(X=4,43 ; d s=0,902)$ \\
\hline Más de 10 años & Cumplir la meta que me trace $(X=4,38 ; d s=0,654)$ \\
\hline \multicolumn{2}{|l|}{ Distancia de carrera } \\
\hline $3 \mathrm{~K}, 5 \mathrm{~K}, 8 \mathrm{~K}$ & Cumplir la meta que me trace $(X=4,35 ; d s=0,609)$ \\
\hline $10 \mathrm{~K}, 21 \mathrm{~K}$ & Competir contra mi propia marca $(\mathrm{X}=3,78 ; \mathrm{d} s=0,756)$ \\
\hline
\end{tabular}

\section{a) Según sexo}

La puntuación más elevada se obtuvo en el ítem cumplir la meta que me trace, con una media de 4,59 (ds=0,758) en damas y $4,48(\mathrm{~d} s=0,827)$ en varones. Entre ambos sexos no existen diferencias significativas $(\mathrm{p}=0,287)$.

Por el contrario, el motivo que menos importancia tiene para ambos géneros es deseo de obtener algún estímulo material con una media de 1,85 para ambos géneros. Estos datos demuestran que ambos géneros se mueven principalmente por los mismos motivos.

\section{b) Según edad}

En relación con la edad se establecieron tres categorías, las cuales presentan la siguiente distribución: un 19,1\% son menores de 18 ańos, un 68,7\% tienen entre 18 y 40 años, mientras que un 12,2\% tiene más de 40 años.

En las tres categorías la puntuación más elevada fue el ítem cumplir la meta que me tracé, donde los menores de 18 años presentan una media de 4,58 (ds=0,724); los competidores de entre 18 y 40 años, 4,52 ( $\mathrm{d} s=0,042)$, y los mayores de 40 años, 4,37 ( $\mathrm{d} s=0,809)$. Entre las tres categorías no existen diferencias significativas $(\mathrm{p}=0,232)$.

El motivo que menos importancia tiene para las tres categorías es deseo de obtener algún estímulo material con una media de 2,16 (ds=1,14) para menores de 18 ańos; $1,89(\mathrm{~d} s=1,07)$ para competidores entre 18 y 40 ańos y 1,98 $(\mathrm{d} s=1,32)$ para mayores de 40 ańos. La prueba de ANOVA revela que no existen diferencias entre las tres categorías $(p=0,109)$.

\section{c) Según nivel de escolaridad}

Para el análisis de esta variable, sólo serán consideradas las categorías de estudios medios y superiores. Para ambas el principal motivo es cumplir la meta que me tracé, donde las personas con estudios secundarios presentan una media de 4,59 $(\mathrm{d} s=0,751)$, mientras aquellas que tienen estudios superiores tienen una media de 4,45 ( $\mathrm{d} s=0,837)$. Los análisis muestran que no existen diferencias entre ambos grupos $(\mathrm{p}=0,072)$.

Como motivo menos importante aparece deseo de obtener algún estímulo material, con una media de 2,08 ( $\mathrm{ds=1,22)}$ para corredores con enseńanza media y $1,84(\mathrm{~d} s=1,04)$ para participantes con educación superior. Entre ambos grupos, el primero presenta puntuaciones más elevadas y estadísticamente significativas $(p=0,034)$.

\section{d) Según experiencia en competencias}

En relación con la participación anterior en otras competencias, un 55,6\% expresó que sí tiene experiencias en competencias, mientras que el $44,4 \%$ no posee experiencia competitiva. En este estudio se observa que la principal razón para todos los grupos es cumplir la meta que me tracé, con una media de $4,51(\mathrm{~d} s=0,720)$ en el caso de las corredores con 1 a 3 años de experiencia, de 4,43(ds=0,902) en corredores con 3 a 10 años de experiencia, de $4,38(\mathrm{ds}=0,654)$ en corredores con más de 10 ańos de experiencia y de 4,36 (ds=0,934) en el de los competidores sin experiencia previa. No existen diferencias significativas entre todos los grupos ( $\mathrm{p}=0,068)$.

Como motivo menos importante aparece deseo de obtener 
algún estímulo material, con una media de $1,95(\mathrm{ds}=1,10)$ para corredores con 1 a 3 ańos de experiencia en competiciones, 2,01 ( $\mathrm{d} s=1,08)$ para corredores con 3 a 10 años de experiencia, 1,89 $(\mathrm{d} s=1,15)$ para corredores con más de 10 años de experiencia y un media de $1,82(\mathrm{~d} s=1,07)$ para participantes sin experiencia. Entre todos los grupos no existen diferencias significativas $(\mathrm{p}=0,214)$.

\section{e) Según distancia de la corrida}

Tomando en cuenta las diferentes distancias de corrida, se observa el motivo cumplir la meta que me tracé como la más importante en corredores de $3 \mathrm{~K}, 5 \mathrm{~K}$ y $8 \mathrm{~K}$. En cambio el motivo competir contra mi propia marca resulta ser la mejor puntuada en corredores de $10 \mathrm{~K}$ y $21 \mathrm{~K}$ (tabla 2 ).

Por último, para todas las corridas, el motivo menos importante es deseo de obtener algún estímulo material.

\section{Discusión y conclusiones}

Los resultados expuestos en el apartado anterior indican que las principales razones que tienen los corredores urbanos en Chile para participar en competencias de resistencia son cumplir la meta que me tracé y competir contra mi propia marca, ambos tienen que ver con las metas de logro personal. En segundo lugar, se ubican los motivos relacionados con la categoría de satisfacción, mientras que el tercer lugar lo ocupa la aptitud fisica. En la investigación española (Llopis y Llopis, 2006), el motivo principal es el placer que se siente al correr, relacionado con la satisfacción, mientras que la motivación de logro se encuentra en segundo lugar. No obstante en el estudio realizado en Cuba por Barrios y Cardozo (2002), el principal motivo es el logro de meta personal, seguido por la comprobación de aptitud física y satisfacción.

Diversos estudios han investigado sobre la motivación en la práctica de actividad física. En uno de ellos, dentro de los motivos principales para practicar ejercicio, están la autosatisfacción, propia imagen y la interdependencia (Ebbeck et al. 1995), motivos asociados al cuarto nivel de las jerarquías de Maslow (1943). Otras investigaciones que adaptaron y validaron cuestionarios al español sobre motivos para practicar ejercicio físico (Moreno et al. 2007, y Capdevilla et al. 2004) arrojaron como resultados motivos relacionados con la salud, bienestar social, apariencia y placer.

Los resultados expuestos en este estudio se relacionan con logros de metas personales de donde se puede inferir que las competencias de resistencia son un lugar que sirve para el desarrollo de la superación y que, a diferencia de otros deportes, aquí la gran mayoría de las personas no corre por competir contra los demás, sino por el contrario, por superarse ellos mismos.

Otro aspecto importante entregado por esta investigación es que la satisfacción personal cumple un rol importante en la motivación para participar en competencias, siendo la segunda categoría de más importancia para los sujetos. Esta categoría es posible relacionarla con el nivel de autorrealización de la pirámide de Maslow. También los sujetos que participan en las competencias buscan evaluar su aptitud física.

En relación a otras variables, Ogles, Masters y Richardson (1995) obtuvieron diferencias en relación al género: mientras que para las mujeres los motivos más importantes son preocupación por el peso, afiliación y autoestima, para los hombres son la motivación de logro y reconocimiento. Por su parte, Pavon (2004, citado en Moreno, Martínez y Alonso, 2006) explica que los motivos relacionados con la salud, liberar energía acumulada y la imagen personal son los principales motivos de practica físico-deportiva de las mujeres, en cambio los hombres la practican por la competición, la auto-superación y las relaciones sociales. Por el contrario de ambos estudios, en esta investigación no se presentan diferencias en relación al género, comprobándose que las principales razones para ambos sexos son la motivación de logro y la satisfacción.

Se ha podido comprobar que, independientemente de las variables, se marca una tendencia general que indica que los principales motivos para competir en carreras de resistencia tienen relación con las metas de logro y la satisfacción, mientras que lo que menos importa es obtener algún premio.

La variabilidad de resultados en los distintos estudios (Moreno et al., 2006; Lores y Murcia, 2008; Salguero y Martos, 2011) refleja que las razones para practicar actividad física dependerán de distintos factores, ya sea el contexto sociocultural en el que se tome la muestra, la actividad física que se practica, el tamaño de la muestra, etc., por lo tanto, los resultados de esta investigación sólo son atribuibles a la realidad estudiada en este caso.

\section{Aplicaciones prácticas}

Los resultados de esta investigación pueden servir como guía que permita desencadenar transformaciones en la forma de abordar algunas políticas relacionadas con el tema, como son políticas preventivas en salud, políticas deportivas, políticas educativas, uso del tiempo libre, etc. De este modo, también se podría mejorar la publicidad, propaganda y difusión para que sean más efectivas, de manera que sea posible generar un mayor ambiente de participación en competencias de resistencia y en actividades físicas en general. 


\section{Referencias bibliográficas}

1. Barrios, R. (2001). Motivación hacia la práctica del ejercicio en corredores cubanos. Disponible en http://www.efdeportes.com/efd31/motiv.htm [visitado el 10 de octubre del 2012].

2. Barrios, R. y Cardozo, L. (2002). Motivación para competir en corredores populares cubanos. Disponible en http://www.efdeportes.com/efd47/ motiv.htm [visitado el 15 octubre del 2012].

3. Buceta, J., López, A., Perez, M., Vallejo, M., \& Del Pino, M. (2002) Intervención psicológica con corredores de maratón: características y valoración del programa aplicado en el maratón de Madrid. Revista de Psicología del Deporte, 11(2), 83-109.

4. Buceta, J., López, A., Perez, M., Vallejo, M., \& Del Pino, M. (2003). Estado psicológico de los corredores populares de maratón en los días anteriores a la prueba. Psicothema, 15(2), 273-277.

5. Cauas, R. (2008). El boom del running en Chile, desde una perspectiva psicológica. Disponible en http://www.efdeportes.com/efd121/el-boomdel-running-en-Chile-desde-una-perspectiva-psicologica.htm [Visitado el 20 Noviembre del 2012].

6. Capdevila, Ll., Niñerola, J., Pintanel, M. (2004). Motivación y actividad física: el autoinforme de motivos para la práctica de ejercicio físico (AMPEF). Revista de Psicología del Deporte, 13(1), 55-74

7. Clough, P., Shepherd, J., Maughan, R. (1989). Los motivos para la participación en la carrera recreativa. Journal of Leisure Research, 21(4), 297-309.

8. Ebbeck, V.,Gibbons, S., Leken-Dahle, L., (1995). Motivos de participación de los adultos en la actividad física: un enfoque interactivo. International Journal of Sport Psychology, 26(2), 262-275.
9. Garita, E. (2006). Motivos de participación y satisfacción en la actividad física, ejercicio físico y el deporte. Revista MH Salud, 3(1), 1.-16.

10. Llopis, D. y Llopis, R. (2006). Razones para participar en carreras de resistencia. Un estudio con corredores aficionados. Cultura, Ciencia y Deporte, 3(4), 33-40.

11. Lores, A.; Murcia, A. (2008). Actitudes de los universitarios ante la práctica físico deportiva: diferencia de géneros. Revista de Psicología del Deporte, 17(1), 7-23.

12. Maslow, A. (1943). Motivación y personalidad. Madrid: Díaz de Santos S.A.

13. Moreno, J., Cervelló, E., Martinez, A. (2007). Validación de la escala de medida de los motivos para la actividad física-revisada en españoles: diferencias por motivos de participación. Anales de Psicología, 23(1), 167-176.

14. Moreno, J.; Martínez, C.; Alonso, N. (2006). Actitudes hacia la práctica físico-deportiva según el sexo del practicante. RICYDE. Revista Internacional de Ciencias del Deporte, 2(3), 20-43.

15. Ogles, B., Masters, K., Richardson, S. (1995). Ejecución obligatoria y género: un análisis de las motivaciones y hábitos participativos de capacitación. International Journal of Sport Psychology, 26(2), 233-248.

16. Salguero, A.; Martios, P. (2011). Desigualdades de género en competiciones populares de fondo. Apunts. Educación Física y Deportes, 103, $91-100$ 